\title{
Does the prevalence of dyslipidemias differ between Newfoundland and the rest of Canada? Findings from the electronic medical records of the Canadian Primary Care Sentinel Surveillance Network
}

\section{Shabnam Asghari ${ }^{1}$, Erfan Aref-Eshghi ${ }^{1}$, Oliver Hurley ${ }^{1}$, Marshall Godwin ${ }^{1}$, Pauline Duke ${ }^{1}$, Tyler Williamson ${ }^{2}$ and Masoud Mahdavian ${ }^{3}$}

1 Memorial University of Newfoundland (MUN), St. John's, NF, Canada

2 University of Calgary, Calgary, $A B$, Canada

${ }^{3}$ University of Ottawa, Ottawa, ON, Canada

Edited by:

Dexter Canoy, University of Oxford,

UK

Reviewed by:

Christoph Sinning, University Heart Center Hamburg, Germany

Lucy Wright, University of Oxford, UK

*Correspondence:

Shabnam Asghari, Primary

Healthcare Research Unit, Faculty of

Medicine, Memorial University of

Newfoundland, 300 Prince Philip

Drive, St. John's, Newfoundland and

Labrador, Canada A1B 3V6

e-mail: shabnam.asghari@med.

mun.ca
Introduction: Newfoundland and Labrador (NL) has the highest prevalence of cardiovascular disease (CVD) in Canada. Dyslipidemia is a risk factor for CVD. This study compares the prevalence of dyslipidemia in the NL population with the rest of Canada.

Methods: A cross-sectional study, using data from the Canadian Primary Care Sentinel Surveillance Network (CPCSSN), was undertaken. The study population included adults, excluding pregnant women, aged 20 years and older. Canadian guidelines were used for classifying dyslipidemia. Univariate and multivariate analyses were conducted to compare the lipid levels and prevalence of dyslipidemia between NL and the rest of Canada.

Results: About 128,825 individuals (NL: 7,772; rest of Canada: 121,053) were identified with a mean age of 59 years (55\% females). Mean levels of total cholesterol (4.96 vs. 4.93, $p=0.03$ ), low-density lipoprotein (LDL) (3.00 vs. $2.90 \mathrm{mmol} / \mathrm{L}, p<0.0001)$, triglyceride (1.47 vs. $1.41 \mathrm{mmol} / \mathrm{L}, p<0.0001)$, and high-density lipoprotein (HDL) $(1.29 \mathrm{vs} .1 .39 \mathrm{mmol} / \mathrm{L}$, $p<0.0001$ ) were significantly different in NL compared to the rest of Canada. Dyslipidemias of LDL ( 29 vs. $25 \% p<0.0001)$, HDL (38 vs. $27 \%, p<0.0001)$, and triglyceride (29 vs. $26 \%, p<0.0001$ ) were significantly more common in NL. After adjustment for confounding variables, NL inhabitants were more likely to have dyslipidemia of total cholesterol (OR: 1.16, 95\% Cl: 1.10-1.23, $p<0.0001$ ), HDL (OR: 1.52, 95\% Cl: 1.44-1.60, $p<0.0001$ ), LDL (OR: $1.38,95 \% \mathrm{Cl}: 1.30-1.46, p<0.0001$ ), and ratio (OR: $1.53,95 \% \mathrm{Cl}: 1.42-1.60$, $p<0.0001)$.

Conclusion:The NL population has a significantly higher rate of dyslipidemia compared to the rest of Canada, and the mean levels of all lipid components are worse in NL. Distinct cultural and genetic features of the NL population may explain this, accounting for a higher rate of CVD in NL.

Keywords: dyslipidemia, Canada, Newfoundland, lipid profile, prevalence, Canadian Primary Care Sentinel Surveillance Network

\section{INTRODUCTION}

Cardiovascular diseases (CVDs) are the leading cause of death worldwide, resulting in more than 17.3 million deaths in 2008 (1). This number is expected to reach 23.3 million in 2030 (2). Geographic variations in cardiovascular mortality have frequently been reported in previous studies. According to the 2008 report by WHO, the age standardized CVD mortality rates in the developed world (Australia, Japan, France, and United States) has been 100-200/100,000 population in contrast to higher rates in the rest of the world (3). The 2014 update on the burden of CVD reports significant regional differences in the age standardized cardiovascular mortality rates across the European continent, including lower rates of 150-180 deaths/100.000 in western European countries (Netherlands, Norway, France, and Switzerland), higher rates among southern countries of Europe such as Greece (246.7/100.000) and Cyprus (219.2/100.000), and rates of above 500/100.000 in eastern European countries (Russia, Romania, and Ukraine) (4).

In Canada, CVD is the main cause of death at $32 \%$ and, after musculoskeletal diseases, has the highest economic burden of disease (5). Compared to other provinces, Newfoundland and Labrador (NL) has the highest level of CVD morbidity and mortality in adults. Between 1995 and 1998 in NL, age standardized CVD rates per 100,000 populations ranged from $320.6 / 100,000$ to $196.9 / 100,000$ (6). During the same period, NL had the highest life expectancy lost (LEL) due to CVD - more than 5.3 years - in all of 
Canada (7). According to a Statistics Canada report in 2007, NL had the highest age standardized mortality rate for major CVDs among all Canadian provinces: $218.5 / 100,000$ population in NL compared to a nationwide average mortality rate of $151.9 / 100,000$ (8). The NL CVD mortality rates are more similar to those of southern European countries as well as low and middle income countries, which have a higher mortality rates compared to the developed world $(3,4)$.

Although regional variations in CVD and CVD morality have been recognized, the explanation for these differences is still unclear. Some studies suggest that these variations could be related to the variations in cardiovascular risk factors including smoking, obesity, hypertension, and dyslipidemia $(9,10)$. Dyslipidemia, defined as abnormal blood lipid levels, includes elevated total cholesterol, low-density lipoprotein (LDL), and triglyceride, and decreased high-density lipoprotein (HDL) is one of the most important and well-known risk factors of CVDs (11). The risk for CVD conferred by dyslipidemia varies according to the type of condition and presence or absence of other risk factors. It has been reported to be one of the most important determinants of myocardial infarctions among adults of young age (12). Dyslipidemia is a multifactorial trait wherein environmental and behavioral factors, as well as genetic predisposition, play a role (13).

Although Canada, in general, has a diversity of cultures and ethnicities, the present NL population almost entirely originates from 20,000 migrants from south-west England and the south of Ireland in the mid-1700s (14). This founding population experienced a low level of in-migration over the centuries, which resulted in NL being one of the few remaining isolated Caucasian populations worldwide (14). This isolation has shaped a unique and homogenous culture and genetic background, both of which may contribute to the prevalence of complex traits such as dyslipidemia and, consequently, CVD (14). It is also notable that there is anecdotal evidence from family physicians in NL, who worked in different provinces that a different pattern of lipid profiles and a higher prevalence of dyslipidemia exists in NL compared to other Canadian provinces.

Dyslipidemia in NL has been previously documented in several reports. School-aged children in the 1980s had higher total cholesterol levels compared to age, sex, and racially matched American children (15). A 1990s' study on the rural Newfoundland population found that $61 \%$ of the subjects had hypercholesterolemia recorded in their medical records (16). A recent study on a sample of 4,424 primary health-care patients in NL reported that $42 \%$ of the patients had high cholesterol, 36\% high LDL, 25\% low HDL, and 25\% abnormal triglyceride; however, the results were not compared with other populations (17). The lack of comparison groups, lack of biochemical measurement, and cohorts, which were not fully representative of the NL population were limitations of these studies. The purpose of the current study is to compare the prevalence of dyslipidemias in residents of NL and compare these with the rest of Canada.

\section{MATERIALS AND METHODS SOURCE OF DATA}

The Canadian Primary Care Sentinel Surveillance Network (CPC$\mathrm{SSN}$ ) is a pan-Canadian network that extracts data from the electronic medical records (EMRs) of family physicians. At the time of this study, the CPCSSN database included data from close to 600 primary care clinicians in rural and urban settings across 10 provinces of Canada. CPCSSN is Canada's first library of digital information based on point-of-care data from primary care practices. Data from these EMRs are extracted quarterly and uploaded in a de-identified format to both regional and central (pan-Canadian) databases. The databases are used for chronic disease surveillance in primary care and are also used as a tool for conducting primary care research (18). At the time of this study, the pan-Canadian CPCSSN database included 844,592 individuals over 20 years of age, corresponding to $3 \%$ of the Canadian population; the NL component included 46,588 individuals, representing $11 \%$ of the NL population (18).

\section{STUDY POPULATION}

All adults over 20 years of age (excluding pregnant women) from the CPCSSN database who had a lipid profile in the CPCSSN database between January 1, 2010 and December 31, 2012 were included in the study. To identify pregnant women, the text and/or ICD code records for every event related to pregnancy were queried (19).

\section{VARIABLES \\ Lipid variables}

Canadian guidelines for the diagnosis and management of dyslipidemia (20), the nationwide protocol for all practitioners in Canada, suggests a lipid screen for all men over 40 years of age, all women of over 50 years, all postmenopausal women, all individuals with diabetes, hypertension, obesity, first degree relative with history of CVD under the age of 60, as well as current smokers. The routine screening test requires the measurement of all lipid components.

In this study, the most recent lipid profiles (total cholesterol, HDL, LDL, and triglyceride) for each individual were recorded. The ratio of total cholesterol to HDL was calculated by dividing total cholesterol by HDL. Dyslipidemia was defined using the Canadian guidelines for the diagnosis and treatment of dyslipidemia (Table 1) (20). Individuals with at least one abnormal component were classified as those with any dyslipidemia.

\section{Geographic variables}

In Canada, a national six-digit postal code is used to identify a geographic location. Each postal code is unique and represents a location in the real world. The first digit is specific to each province, whereas the second one classifies the region as rural or urban. Accordingly, we used the first digit of the postal codes to separate those patients living in NL from those in the rest of Canada, and

Table 1 | Healthy levels of serum lipids for Canadian adults (20-23)

\begin{tabular}{ll}
\hline Total cholesterol & $<5.2 \mathrm{mmol} / \mathrm{L}(20-79$ years $)$ \\
Triglyceride & $<1.7 \mathrm{mmol} / \mathrm{L}$ \\
Low-density lipoprotein-cholesterol (LDL) & $<3.4 \mathrm{mmol} / \mathrm{L}$ \\
High-density lipoprotein-cholesterol (HDL) & $>1.0 \mathrm{mmol} / \mathrm{L} \mathrm{men} ;>1.3 \mathrm{mmol} / \mathrm{L}$ \\
& women \\
Ratio of total cholesterol to HDL & $<5.0$
\end{tabular}


the second digit to classify individuals as rural or urban residents (24). The rural/urban residence was included in the multivariate analysis as previous studies suggest that the CVD risk factors could differ between the rural and urban inhabitants (25).

\section{Covariates}

To account for confounding factors, CVD risk factors as well as other variables with effect on lipid levels were extracted from the EMR (20). The demographic variables included age and gender. Obesity was defined as $\mathrm{BMI} \geq 30$; whereas those with $\mathrm{BMI}$ lower than 30, but higher than 25 were classified as overweight. The smoking status was extracted from the most recent record by the family physician at the time of the lipid test, and individuals were classified as non-smokers, past smokers, and current smokers. CPCSSN algorithms for chronic conditions were used to ascertain both diabetes and hypertension (26). These algorithms have high sensitivity and specificity to detect diabetes and hypertension (26). Diagnostic text and ICD code records related to these conditions in EMRs were used for other chronic conditions, including dyslipidemia and $\operatorname{CVD}(27,28)$. Medication use was identified using the text record of the medication name and/or anatomical therapeutic chemical (ATC) codes. Usage of lipid modifying agents (HMG-CoA reductase inhibitors, fibrates, bile acid sequestrants, nicotinic acid, and other agents) was stratified into three categories: current users (any record of lipid-lowering medication use before the date of a blood test and continuing until up to the time of the blood test); previous users (record of drug use between 2 years and 3 months before the date of the blood test); and non-users (no drug use for the last 2 years before the date of a blood test). The classification of medication use here has been previously used in other studies involving the study of lipid profile databases (29). Medications with unintended effects on lipid levels (30) including thiazides, loop diuretics, beta blockers, alpha blockers, ACE inhibitors, calcium channel blockers, estrogen, progesterone, hormone replacement therapy, and corticosteroids were extracted from EMRs.

\section{STATISTICAL ANALYSIS}

Characteristics of the study population, as well as the mean and confidence intervals of the individual lipid components, were summarized using descriptive statistics. Classical tests of hypothesis including Student's $t$-test and the chi-squared test were conducted to test for the association between variables. Logistic regression modeling was used to examine the association between dyslipidemia and living in NL while controlling for age, sex, rurality, and other potential influential factors. For variables with more than $5 \%$ of missing information, i.e., smoking ( $70 \%$ missing $)$ and BMI ( $\sim 50 \%$ missing), a code for missing values was considered wherever model based analyses were performed. In the model, age was classified into three groups and each group was compared against the oldest one (Age $>65$ ); smoking variables were compared with non-smokers; overweight and obese patients were compared with normal and underweight individuals; non-medication users and previous medication users were compared with current users as baseline. A subgroup analysis was also performed to investigate how the patients of EMR primary care in NL differ from the patients of EMR primary care in the rest of Atlantic Canada. A $p$-value $<0.05$ was considered statistically significant. All analyses were performed using STATA/SE 11.2 (Stata Corp., College Station, TX, USA). Geographical variation in the prevalence of dyslipidemia was also presented using ArcMap 10.0 (Build 4000).

\section{ETHICS}

The study protocol was approved for ethics by Health Research Ethics Authority (HREA) of Newfoundland and Labrador.

\section{RESULTS \\ POPULATION DESCRIPTION}

Among the 430,169 individuals recorded in the CPCSSN database from January 1, 2010 to December 31, 2012, 128,825 individuals $(\sim 30 \%)$ had completed blood testing and met the study criteria. The mean age was 59 years, $55 \%$ were women, and $77 \%$ were living in an urban area. The majority of the population $(78 \%)$ was not taking any lipid-lowering medications, whereas 8 and $14 \%$ were categorized as previous users and current users, respectively. Among the drug users, HMG-CoA reductases (Statins) were the most commonly used form of lipid-lowering drugs (94\%), while other drug consumption was rare (data not shown). Table 2 presents general characteristics of the study population in NL and the rest of Canada.

\section{COMPARISON OF LIPID LEVELS AND DYSLIPIDEMIA IN NL WITH THE REST OF CANADA}

Mean levels for all lipid components in NL and the rest of Canada are presented in Table 3. There are significant differences between NL and the rest of Canada for all of the lipid components. Approximately $72 \%$ of the NL population have at least one abnormal lipid component (any dyslipidemia), whereas this figure is $64 \%$ in the rest of Canada $(p<0.0001)$. Figure 1 illustrates the prevalence of dyslipidemia in NL and the rest of Canada. Prevalence of abnormal

\begin{tabular}{|c|c|c|c|}
\hline Characteristics & $\begin{array}{l}\text { Newfoundland } \\
\text { and Labrador (\%) } \\
(n=7,772)\end{array}$ & $\begin{array}{c}\text { Rest of } \\
\text { Canada (\%) } \\
(n=121,053)\end{array}$ & $p$-value \\
\hline Age $[($ mean $(95 \% \mathrm{Cl})]$ & $58.3(58.0-58.6)$ & $59.2(59.1-59.3)$ & $<0.0001$ \\
\hline $\begin{array}{l}\text { Body mass index [(mean } \\
(95 \% \mathrm{Cl})]\end{array}$ & $30.3(29.8-30.9)$ & $28.1(28.0-28.1)$ & $<0.0001$ \\
\hline Sex (female) & 55.8 & 50.1 & 0.70 \\
\hline Residence (rural) & 25.1 & 22.6 & $<0.0001$ \\
\hline Smoking (current) & 22.4 & 13.5 & $<0.0001$ \\
\hline Smoking (past) & 22.1 & 42.8 & $<0.0001$ \\
\hline Hypertension & 33.5 & 34.0 & 0.39 \\
\hline Diabetes & 16.2 & 15.3 & 0.04 \\
\hline History of dyslipidemia & 39.9 & 20.7 & $<0.0001$ \\
\hline Cardiovascular disease & 44.9 & 35.4 & $<0.0001$ \\
\hline $\begin{array}{l}\text { Lipid-lowering medication } \\
\text { (current users) }\end{array}$ & 20.5 & 13.6 & $<0.0001$ \\
\hline $\begin{array}{l}\text { Lipid-lowering medication } \\
\text { (previous users) }\end{array}$ & 8.0 & 8.0 & 0.95 \\
\hline
\end{tabular}

$\mathrm{Cl}$, confidence interval. 
Table 3 | Mean levels of lipid components in Newfoundland and Labrador and the rest of Canada [mean (95\% confidence interval)].

\begin{tabular}{lccc}
\hline & $\begin{array}{l}\text { Newfoundland } \\
\text { and Labrador }\end{array}$ & Rest of Canada & $\begin{array}{c}\boldsymbol{p} \text {-value } \\
(\boldsymbol{t} \text {-test) }\end{array}$ \\
\hline Total cholesterol & $4.96(4.94-4.98)$ & $4.93(4.93-4.94)$ & 0.034 \\
Low-density lipoprotein & $3.00(2.98-3.02)$ & $2.90(2.89-2.91)$ & $<0.0001$ \\
High-density lipoprotein & $1.29(1.28-1.30)$ & $1.39(1.39-1.40)$ & $<0.0001$ \\
Triglyceride & $1.47(1.45-1.49)$ & $1.41(1.40-1.42)$ & $<0.0001$ \\
Ratio & $4.06(4.04-4.09)$ & $3.76(3.75-3.77)$ & $<0.0001$
\end{tabular}

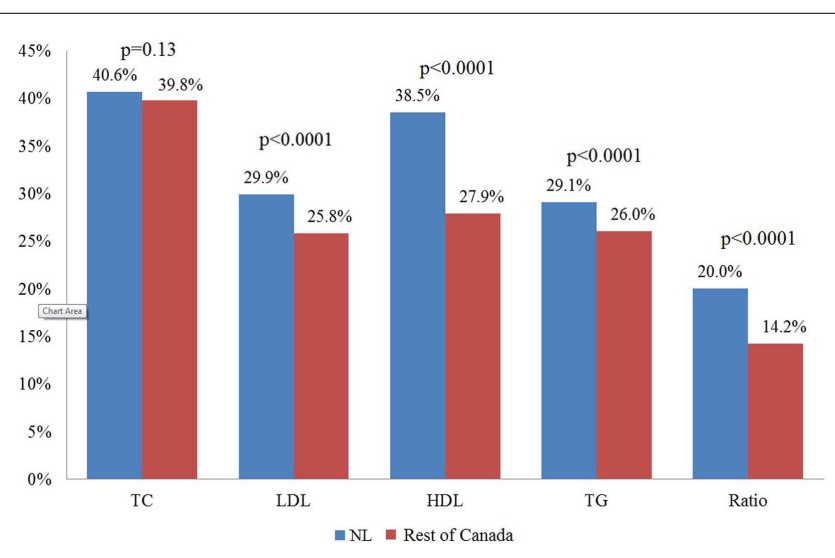

FIGURE 1 | Percentage of adults with unhealthy levels of lipids in patients of primary care in Newfoundland and Labrador and the rest of Canada. NL, Newfoundland and Labrador

LDL (29 vs. $25 \%$ ), HDL (38 vs. $27 \%$ ), triglyceride (29 vs. $26 \%$ ), and ratio (20 vs. $14 \%)$ were significantly higher in NL $(p<0.0001)$. The greatest difference was observed for HDL dyslipidemia with an $11 \%$ difference (Figures $\mathbf{1}$ and $\mathbf{3}$ ).

\section{COMPARISON OF DYSLIPIDEMIA IN NL WITH REST OF CANADA CONTROLLING FOR COVARIATES}

Table 4 shows the results of the multivariate logistic regression model for dyslipidemia. After adjusting for the effect of covariates, NL inhabitants were more likely to have dyslipidemia of total cholesterol (OR: 1.16, 95\% CI: 1.10-1.23, $p<0.0001$ ), HDL (OR: 1.52, 95\% CI: $1.44-1.60, p<0.0001$ ), LDL (OR: $1.38,95 \% \mathrm{CI}$ : $1.30-1.46, p<0.0001$ ), and total cholesterol/HDL ratio (OR: 1.53, 95\% CI: $1.42-1.60, p<0.0001)$.

High-density lipoprotein dyslipidemia was significantly more common in adults <40 years old (OR: 2.10, 95\%CI: 1.99-2.23, $p<0.0001)$ compared to other age groups. The greatest effect on the dyslipidemias of all components was observed by lipidlowering medications (except for HDL and triglyceride) followed by age and body mass index. Drugs with unintended lipid effects had no significant influence on lipid levels. Further analysis on the lipid levels was performed using linear regression modeling and findings with similar trends were observed (data not shown).

\section{COMPARISON OF DYSLIPIDEMIA BETWEEN NL AND ATLANTIC CANADA}

Our data identified 25,409 individuals from Atlantic Canada, New Brunswick, Nova Scotia, Prince Edward, and NL. The NL population had significantly higher levels of total cholesterol (4.96 vs. $4.83, p<0.0001)$, LDL (3.00 vs. $2.88, p<0.0001)$, and total cholesterol/HDL ratio ( 4.06 vs. $3.99, p=0.04$ ) compared to the rest of Atlantic Canada. No significant difference was observed between NL and the other Atlantic Canadian provinces for triglyceride and HDL levels.

Compared to the rest of Atlantic Canada, the NL population had a higher prevalence of total cholesterol dyslipidemia (40 vs. $37 \%, p<0.0001$ ), HDL dyslipidemia (38 vs. $37 \%, p=0.04$ ), LDL dyslipidemia ( 30 vs. $27 \%, p<0.0001$ ), and non-significant trends toward higher triglyceride dyslipidemia, and ratio dyslipidemia. The results did not change after adjustment for covariates using multivariate logistic regression (data not shown). Distribution of those with any dyslipidemia as well as HDL dyslipidemia in these patients in NL and the four Canadian regions including Atlantic Canada, Central Canada, Prairies, and Pacific Canada are illustrated in Figures 2 and 3, respectively.

\section{DISCUSSION}

Our study included a large population of individuals who visited family physicians in NL and across Canada. To our knowledge, this is the first study comparing biochemical measurements of lipid profiles between two large populations from NL and the rest of Canada. The prevalence of dyslipidemia in NL is significantly higher compared to the rest of Canada with the greatest difference found for HDL dyslipidemia. This might suggest that the actual differences in dyslipidemia are much higher than what was observed in our study. HDL levels are not generally influenced by the common therapeutic agents administered for the management of dyslipidemia (21). This is also consistent with the finding from multivariate models in our study where a weak association is reported between lipid-lowering medications and HDL. Moreover, the adults in our study who were younger than 40 years of age were more likely to have lower HDL level compared to the adults of other age groups. Although this might be related to the possibility that the younger individuals in our study are from a high risk group, further investigation of the associated factors of lower HDL level among this age group could be an area of concentration for future studies.

This study has some limitations. It is a secondary analysis of EMR data from primary care practices in Canada. EMR data are collected for clinical practice and the extent to which this data could be accurate for research could be questioned. Furthermore, we used a cross-sectional study design, which meant to provide a snapshot of dyslipidemia in Canada. The casual/temporal relationship between lipid abnormalities and their risk factors should be interpreted with caution. The negative association between dyslipidemia and morbidities including CVD, diabetes, and hypertension should be interpreted in the line of a therapeutic regime suggested by the CVD prevention guidelines for patients with those conditions (20).

Electronic medical records are becoming more commonly used in medical practice; however, the quality of EMR data may not be optimal. A systematic review on use of EMR for health outcome research suggests that the validity of EMR data differs from country to country and from health condition to health condition (31). A recent study by $\mathrm{Tu}$ et al. assessed the completeness of primary 


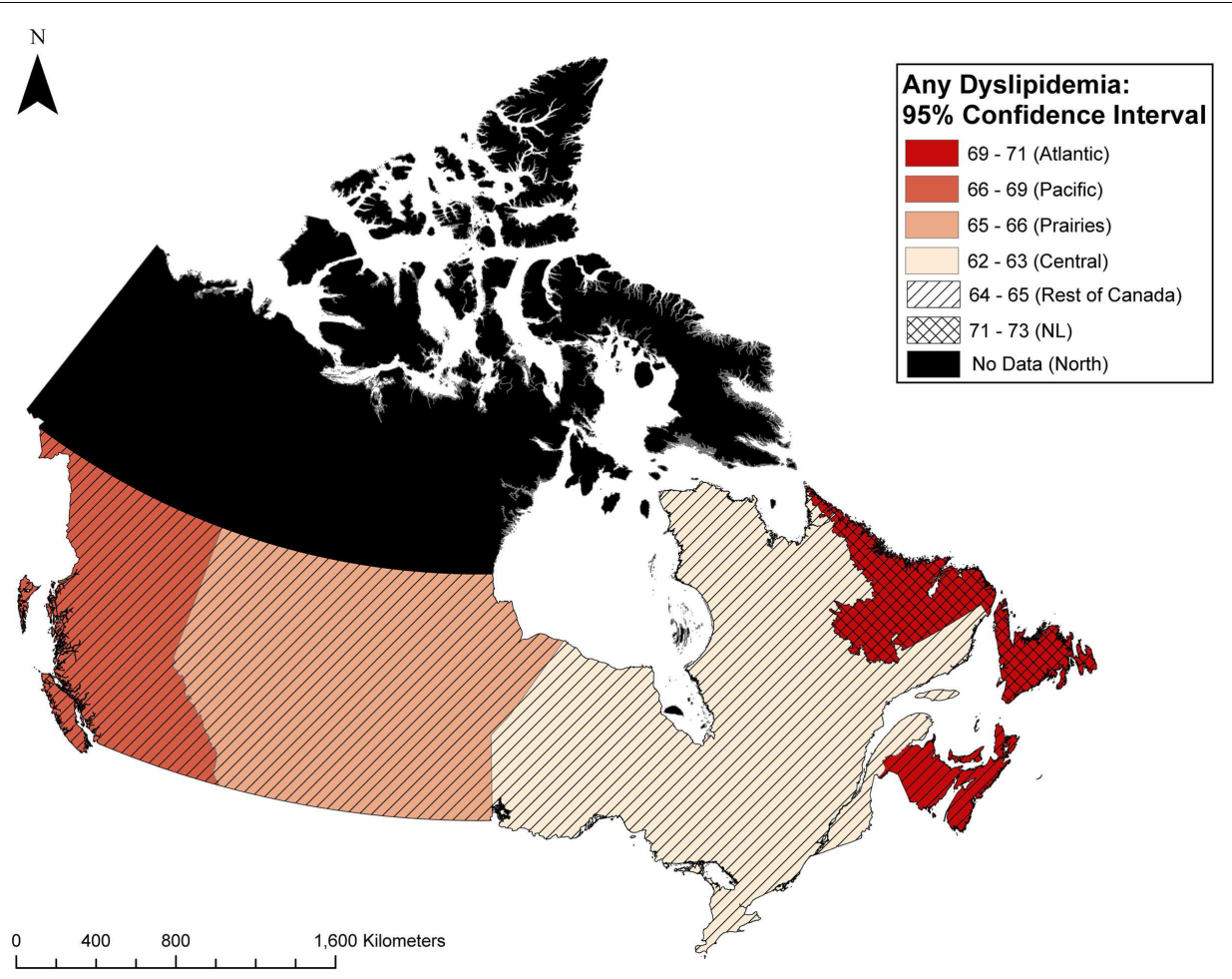

FIGURE 2 | Geographic distribution of those with any dyslipidemia in primary care patients in Canada.

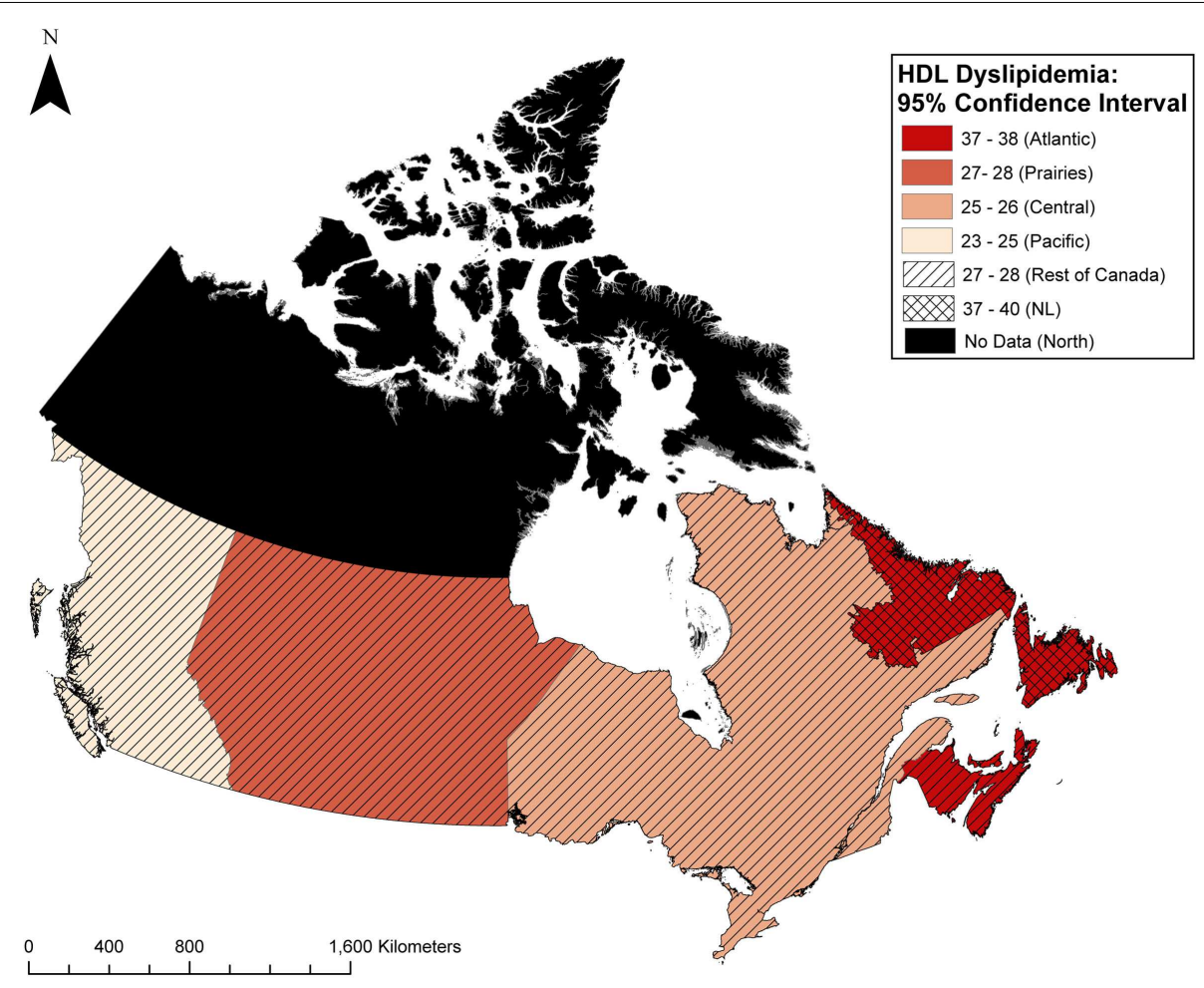

FIGURE 3 | Geographic distribution of HDL dyslipidemia in primary care patients in Canada. 
Table 4 | Multivariable logistic regression models assessing lipid abnormalities between Newfoundland and Labrador compared to the rest of Canada [odds ratio $(95 \%$ confidence interval)].

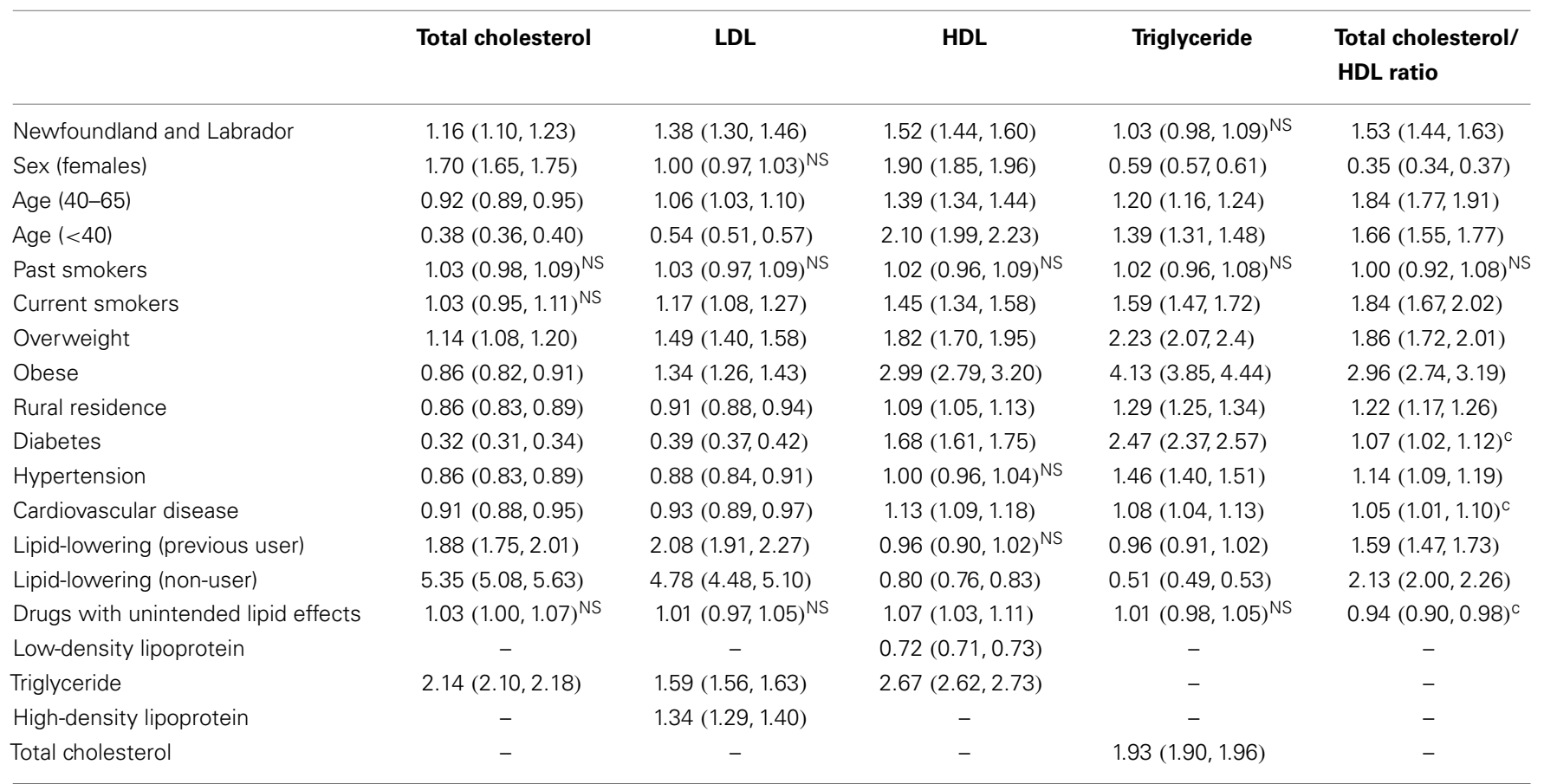

All the presented odds ratios are significant at $p<0.0001$ except for ${ }^{c} p<0.05,{ }^{\text {Ns }}$ not significant $(p>0.05)$.

care EMR data in Ontario, Canada. The study shows good capture of information and low level of missing information within primary care EMRs compared with administrative data (32). Another study using data from CPCSSN shows high validity and reliability for eight common chronic conditions in primary care CPCSSN data. The study suggests that CPCSSN data are a suitable source for health service research (33).

Statistics Canada surveys could be a complement to the EMR data that we used in the study, as the best measure for examining the discrepancies in lipid profiles between different communities and geographic regions. Especially, if it is argued that our results only correspond to a portion of the population who had a lipid profile conducted by their family physician; generally speaking, a population with higher morbidity.

This study, however, is the first of its kind to compare a large population from NL and the rest of Canada (11 and 3\% of the overall population, respectively), and the findings show strong similarities with lipid levels of Canadians as reported from the Canadian Health Measure Surveys (22, 23, 34, 35), in serum levels of total cholesterol (4.94 vs. 4.93), LDL (2.91 vs. 2.90), HDL (1.39 vs. 1.41$)$, triglyceride (1.42 vs. 1.35 ), and ratio (3.79 vs. 3.77 ) as well as the prevalence of dyslipidemia (22) of total cholesterol (both $40 \%$ ), LDL (both $26 \%$ ), HDL (28 vs. $25 \%$ ), and ratio (14 vs. $16 \%$ ), respectively. Findings from our previous study in NL, using secondary data from NL laboratories on a sample of 94,000 adults 20 years and older who had lipid profile tests between 2009 and 2010, also showed similarities with the present study (36). Both of these reports support differences in lipid levels and the higher prevalence of dyslipidemia in NL, particularly for HDL dyslipidemia.

\section{IMPLICATIONS FOR CLINICAL PRACTICE}

The fundamental research idea came from experienced clinicians in several Canadian provinces who suspected an abnormality in Newfoundlanders' lipid profiles. Our results are in agreement with their speculations. The observed differences in the prevalence of dyslipidemia in our study will have clinical benefits in designing guidelines for the treatment and prevention of dyslipidemia and CVD, as well as in identifying the contributing factors in a distinct population such as NL, where the current therapeutic and prevention guidelines might not be as effective and applicable. This is particularly of importance for HDL-C, which is known to be a key determinant of CVD risks, which even persists among patients with low levels of LDL-C (37).

The findings in our study are also consistent with previous studies suggesting regional differences in the prevalence of dyslipidemia in different regions of Europe (38) and among Hispanic and non-Hispanic whites populations in USA (39). These studies suggested a more aggressive management of the risk factors in the afflicted areas. Such a strategy could also be considered by the authorities in NL province of Canada.

\section{CONCLUSION}

Analyses of the CPSSN data indicate a significantly higher prevalence of adverse lipid components in NL compared to the rest of Canada, including a higher prevalence of low HDL in NL.

It is difficult to determine exactly what the lower HDL levels in younger adults and unhealthy levels of lipids in adults generally mean for CVD prevalence in the future as the individuals age; however, it may be an indication that the high rate of CVD in NL is not likely to change any time soon. The results of this study provide 
more evidence of our theory that a different pattern of serum lipids is present in NL, which has a homogenous population, and suggests that more research into lipid profiles is required, especially with regard to the effects of HDL on cardiovascular health in that population. Our findings will be beneficial in designing guidelines for the treatment and prevention of dyslipidemia and $\mathrm{CVD}$, as well as in identifying the contributing factors specific to NL population.

\section{ACKNOWLEDGMENTS}

The authors acknowledge Ms. Kathleen Murphy for language editing of the manuscript.

\section{REFERENCES}

1. World Health Organization (2011). Global Status Report on Noncommunicable Diseases 2010. Available from: http://whqlibdoc.who.int/publications/2011/ 9789240686458_eng.pdf?ua=1

2. Mathers CD, Loncar D. Projections of global mortality and burden of disease from 2002 to 2030. PLoS Med (2006) 3(11):e442. doi:10.1371/journal.pmed. 0030442

3. WHO. The Global Burden of Disease. Geneva: World Health Organization (2008).

4. Melanie N, Nick T, Peter S, Mike R. Cardiovascular disease in Europe 2014: epidemiological update. Eur Heart J (2014) 35(42):2950-9. doi:10.1093/eurheartj/ ehu299

5. Public Health Agency of Canada, Canadian Institute for Health Information, Heart and Stroke Foundation of Canada, Statistics Canada. Tracking Heart Disease and Stroke in Canada (2009). Available from: http://www.phac-aspc.gc.ca/ publicat/2009/cvd-avc/pdf/cvd-avs-2009-eng.pdf

6. Filate WA, Johansen HL, Kennedy CC, Tu JV. Regional variations in cardiovascular mortality in Canada. Can J Cardiol (2003) 19(11):1241-8.

7. Manuel DG, Leung M, Nguyen K, Tanuseputro P, Johansen K. Burden of cardiovascular disease in Canada. Can J Cardiol (2003) 19(9):997-1004.

8. Statistics Canada. Mortality, Summary List of Causes (2007). Available from: http://www.statcan.gc.ca/pub/84f0209x/84f0209x2007000-eng.htm

9. Jenum AK, Stensvold I, Thelle DS. Differences in cardiovascular disease mortality and major risk factors between districts in Oslo; An ecological analysis. Int J Epidemiol (2001) 30(Suppl 1):S59. doi:10.1093/ije/30.suppl_1.S59

10. Gupta R, Guptha S, Sharma KK, Gupta A, Deedwania P. Regional variations in cardiovascular risk factors in India: India heart watch. World J Cardiol (2012) 4(4):112. doi:10.4330/wjc.v4.i4.112

11. Musunuru K. Atherogenic dyslipidemia: cardiovascular risk and dietary intervention. Lipids (2010) 45(10):907-14. doi:10.1007/s11745-010-3408-1

12. Faisal AW, Ayub M, Waseem T, Khan RS, Hasnain SS. Risk factors in young patients of acute myocardial infarction. J Ayub Med Coll Abbottabad (2011) 23(3):10-3.

13. Schaefer EJ. Diagnosis and management of lipoprotein disorders. In: Rifkind BM, editor. Drug Treatment of Hyperlipidemia. New York, NY: Dekker (1991). p. 17-52.

14. Rahman P, Jones A, Curtis J, Bartlett S, Peddle L, Fernandez BA, et al. The Newfoundland population: a unique resource for genetic investigation of complex diseases. Hum Mol Genet (2003) 12:R167-72. doi:10.1093/hmg/ddg257

15. Fodor JG, Balram BC, Heyden S. Lipid and lipoprotein levels of Newfoundland school children. Ann Nutr Metab (1987) 31(4):231-6. doi:10.1159/ 000177273

16. Chockalingam A, Fodor JG. Cardiovascular risk factors in Newfoundland population and modification of their level through nonpharmacological intervention. J Cardiovasc Pharmacol (1990) 16(Suppl 8):S54-6. doi:10.1097/00005344199006168-00021

17. Justin O, Shabnam A, Marshall G, Kayla C, Kris A. Prevalence of dyslipidemia in Newfoundland adults: approaches to estimation using electronic medical records. J Epidemiol Community Health (2013) 67:e1. doi:10.1136/jech-2013202386.4

18. Birtwhistle R, Keshavjee K, Lambert-Lanning A, Godwin M, Greiver M, Manca D, et al. Building a pan-Canadian primary care sentinel surveillance network: initial development and moving forward. J Am Board Fam Med (2009) 22(4):412-22. doi:10.3122/jabfm.2009.04.090081
19. Devine S, West S, Andrews E, Tennis P, Hammad TA, Eaton S, et al. The identification of pregnancies within the general practice research database. Pharmacoepidemiol Drug Saf (2010) 19(1):45-50. doi:10.1002/pds.1862

20. Genest J, McPherson R, Frohlich J, Anderson T, Campbell N, Carpentier A, et al. Canadian cardiovascular society/Canadian guidelines for the diagnosis and treatment of dyslipidemia and prevention of cardiovascular disease in the adult - 2009 recommendations. Can J Cardiol (2009) 25(10):567-79. doi:10.1016/S0828-282X(09)70715-9

21. Cannon CP. High-density lipoprotein cholesterol as the Holy Grail. JAMA (2011) 306(19):2153-5. doi:10.1001/jama.2011.1687

22. Statistics Canada. Canadian Health Measures Survey - Cholesterol Levels of Canadians, 2009 to 2011 (2014). Available from: http://www.statcan.gc.ca/pub/ 82-625-x/2012001/article/11732-eng.htm

23. Heart Health and Cholesterol Levels of Canadians, 2007 to 2009; Component of Statistics Canada Catalogue no. 82-625-X Health Fact Sheets. (2010). Available from: http://www.statcan.gc.ca/pub/82-625-x/2010001/article/11136eng.pdf

24. Population and Dwelling Counts, for Canada, Census Metropolitan Areas, Census Agglomerations and Census Subdivisions (Municipalities), 2011 and 2006 Censuses. Statistics Canada (2014). Available from: http://www12. statcan.gc.ca/census-recensement/2011/dp-pd/hlt-fst/pd-pl/Table-Tableau. cfm?LANG=Eng\&T $=203 \& \mathrm{CMA}=001 \& \mathrm{~S}=0$ \&O$=\mathrm{D} \& \mathrm{RPP}=25$

25. Lindroth M, Lundqvist R, Lilja M, Eliasson M. Cardiovascular risk factors differ between rural and urban Sweden: the 2009 Northern Sweden MONICA cohort. BMC Public Health (2014) 14(1):825. doi:10.1186/1471-2458-14-825

26. Kadhim-Saleh A, Green M, Williamson T, Hunter D, Birtwhistle R. Validation of the diagnostic algorithms for 5 chronic conditions in the Canadian primary care sentinel surveillance network (CPCSSN): a Kingston practice-based research network (PBRN) report. J Am Board Fam Med (2013) 26(2):159-67. doi:10.3122/jabfm.2013.02.120183

27. Herrett E, Thomas SL, Schoonen WM, Smeeth L, Hall AJ. Validation and validity of diagnoses in the general practice research database: a systematic review. Br J Clin Pharmacol (2010) 69(1):4-14. doi:10.1111/j.1365-2125.2009. 03537.x

28. Gulliford MC, Charlton J, Ashworth M, Rudd AG, Toschke AM, eCRT Research Team. Selection of medical diagnostic codes for analysis of electronic patient records. Application to stroke in a primary care database. PLoS One (2009) 4(9):e7168. doi:10.1371/journal.pone.0007168

29. Jones PH, Nair R, Thakker KM. Prevalence of dyslipidemia and lipid goal attainment in statin-treated subjects from 3 data sources: a retrospective analysis. J Am Heart Assoc (2012) 1:e001800. doi:10.1161/JAHA.112.001800

30. Mantel-Teeuwisse AK, Kloosterman JM, Maitland-van der Zee AH, Klungel OH, Porsuis AJ, de Boer A. Drug-induced lipid changes: a review of the unintended effects of some commonly used drugs on serum lipid levels. Drug Safety (2001) 24(6):433-56. doi:10.2165/00002018-200124060-00003

31. Chan KS, Fowles JB, Weiner JP. Electronic health records and reliability and validity of quality measures: a review of the literature. Med Care Res Rev (2010) 67(5):503-27. doi:10.1177/1077558709359007

32. Tu K, Mitiku TF, Ivers NM, Guo H, Lu H, Jaakkimainen L, et al. Evaluation of electronic medical record administrative data linked database (EMRALD). Am JManag Care (2014) 20(1):e15-21.

33. Williamson T, Green ME, Birtwhistle R, Khan S, Garies S, Wong ST, et al. Validating the $8 \mathrm{CPCSSN}$ case definitions for chronic disease surveillance in a primary care database of electronic health records. Ann Fam Med (2014) 12(4):367-72. doi:10.1370/afm.1644

34. Statistics Canada. Canadian Health Measures Survey - Cholesterol Levels of Canadians, Cycle 2 Data; 2009 to 2011 (2014). Available from: http://www.statcan.gc. $\mathrm{ca} / \mathrm{pub} / 82-626-\mathrm{x} / 82-626-\mathrm{x} 2013001-\mathrm{eng} . \mathrm{pdf}$

35. Statistics Canada. Canadian Health Measures Survey-Cholesterol Levels of Canadians, Cycle 2 Data; 2007 to 2009 (2014). Available from: http://www.statcan.gc. ca/pub/82-623-x/2010002/t055-eng.htm

36. Lee S, Godwin M, Aubrey K, Collins K, Duke P, Asghari S (2012). Rural-Urban Differences in Prevalence of Dyslipidemia in Newfoundland: Finding from Eastern Health Laboratory Information System. St. John's, NF: Primary Care Partnership Forum.

37. Leeper NJ, Heidenreich PA. Clinical significance of high-density lipoprotein cholesterol in patients with low low-density lipoprotein cholesterol. J Am Coll Cardiol (2008) 51(1):49-55. doi:10.1016/j.jacc.2007.07.086 
38. Farsang C, Naditch-Brule L, Perlini S, Zidek W, Kjeldsen SE, GOOD investigators. Inter-regional comparisons of the prevalence of cardiometabolic risk factors in patients with hypertension in Europe: the GOOD survey. J Hum Hypertens (2009) 23(5):316-24. doi:10.1038/jhh.2008.136

39. Hertz RP, Unger AN, Ferrario CM. Diabetes, hypertension, and dyslipidemia in Mexican Americans and non-Hispanic whites. Am J Prev Med (2006) 30(2):103-10. doi:10.1016/j.amepre.2005.10.015

Conflict of Interest Statement: The authors declare that the research was conducted in the absence of any commercial or financial relationships that could be construed as a potential conflict of interest.

Received: 08 October 2014; accepted: 03 January 2015; published online: 02 February 2015 .
Citation: Asghari S, Aref-Eshghi E, Hurley O, Godwin M, Duke P, Williamson T and Mahdavian M (2015) Does the prevalence of dyslipidemias differ between Newfoundland and the rest of Canada? Findings from the electronic medical records of the Canadian Primary Care Sentinel Surveillance Network. Front. Cardiovasc. Med. 2:1. doi: $10.3389 / f_{c} v m .2015 .00001$

This article was submitted to Cardiovascular Epidemiology, a section of the journal Frontiers in Cardiovascular Medicine.

Copyright (C) 2015 Asghari, Aref-Eshghi, Hurley, Godwin, Duke, Williamson and Mahdavian. This is an open-access article distributed under the terms of the Creative Commons Attribution License (CC BY). The use, distribution or reproduction in other forums is permitted, provided the original author(s) or licensor are credited and that the original publication in this journal is cited, in accordance with accepted academic practice. No use, distribution or reproduction is permitted which does not comply with these terms. 